various non-pharmacological/psychological interventions in the management of CYP with ADHD.

Results Non-Pharmacological/psychological treatment modalities can be classified into 4 major categories including Psychosocial (eg social skills and parent training), Body-focused (eg yoga and physical exercise), Cognitive/neuro-Cognitive Therapy (CT): (eg computer attention training and Neurofeedback) and Cognitive-behavioural interventions (eg Psychoeducation and play therapy).

Published literature provides variable level of evidence for the beneficial effects of non-Pharmacological interventions on important aspects of child and parent functioning. Combination of psychological therapy (including group-based parental Psychoeducation) with psychostimulants has the greatest effect size for controlling the core and other ADHD-related symptoms compared to either psychostimulants or behavioural therapies alone. There is currently insufficient evidence to recommend standalone Behavioural therapy or Cognitive behaviour therapy, working memory or Neurocognitive training and Neurofeedback for controlling core symptoms of ADHD.

There is strong evidence for parent training and other adult/child-based behavioural interventions in controlling ADHD-related outcomes such as parenting skills, child's social skills, academic performance and challenging behaviours. Research has also proven the effectiveness of classroom-based psychosocial interventions for reducing disruptive behaviours, while use of Computer-based attention and other Neurocognitive training, as well as participation in regular Cardio exercises show moderate evidence for improving Executive skills.

On the other hand, combinations of different types of nonpharmacological modalities have disappointing low efficacy in the management of both the core symptoms and other related outcomes in ADHD.

Conclusions A wide range of non-pharmacological/behavioural interventions have been recommended as part of a Multimodal management strategy for CYP with ADHD. The significant importance of non-pharmacological interventions for ADHD has become more relevant recently, especially during the COVD 19 pandemic crisis. All clinicians need to be familiar with the rationale and principles of different modalities of non-pharmacological interventions and be confident in offering them through 'social prescribing', by signposting to various local providers or recommending self-directed access to several free or commercial online resources.

\section{CHANGES TO THE INITIAL HEALTH ASSESSMENT (IHA) FOR CHILDREN ENTERING THE LOCAL AUTHORITY CARE SYSTEM DURING COVID-19 PANDEMIC CRISIS}

\begin{abstract}
${ }^{1}$ Christine Muirden, ${ }^{2}$ Anita Root, ${ }^{3}$ Joy Edwards, ${ }^{4}$ Georgia Edwards, ${ }^{5}$ Amin Band, ${ }^{6}$ Hani Ayyash. ${ }^{1}$ West Essex CCG; ${ }^{2}$ West Essex Clinical Commissioning Group, Spencer Close, Epping; ${ }^{3}$, Mid Essex Clinical Commissioning Group, Wren House, Hedgerows Business Park, Colchester Road, Chelmsford; ${ }^{4}$ North East Essex Clinical Commissioning Group, Aspen House, Stephenson Road, Severalls Business Park, Colchester, ${ }^{5}$ Provide, Aspen House, Stephenson Road, Severalls Business Park, Colchester; ${ }^{6}$ Integrated Department of Paediatrics Mid and South Essex University Hospitals Group, Southend University Hospital NHS Foundation Trust, Prittlewell Chase, Westcliff-on- Sea
\end{abstract}

\subsection{6/archdischild-2021-rcpch.861}

Background Covid-19 has impacted on the healthcare provision worldwide. Anecdotally, assessment and management of looked after children have been restricted, resulting in assessment being carried out virtually by telephone or videoconsultation. While changes to traditional face to face appointments were necessary enabling consultations to continue, it was unclear whether new technologies would be reliable or effective for vulnerable children.

Objectives

1. To review the decision making process which informed the method for undertaking an Initial Health Assessment (IHA) for children during the Covid-19 pandemic

2. To determine how any gaps were mitigated within the child's health care plan.

Methods Following consent from individual organisations, 56 anonymised IHA records were randomly selected to be reviewed against an audit template from each provider across Southend, Essex and Thurrock (SET). The timeframe for the assessment was from 1st April 2020 until 30th September 2020. The data was measured against IHA pathway standards, a guide to support decision making. A table of comparative data was mapped to provide the basis for analysis.

Results Of the five SET service providers invited to participate, four organisations provided a response within the timeframe. A total of 21 questionnaires were received. The cohort consisted of 9 females and 12 males. Ages ranged from 3 weeks to 17 years. One review was face-to-face, 4 were via video, 6 by telephone, and a further 10 recorded as virtual (unknown whether assessment was by telephone or by video-consultation). Of 11 children with no previous health review, 6 were sign-posted to the GP for a physical assessment. Similarly, 6 of the 9 children with a previous medical contact were referred to a GP. Eight children had no recorded physical follow-up.

Conclusions It is important to recognise the inclusive data provided just a snapshot of the IHAs undertaken during this period. However, it was evident that virtual assessments appeared to become the default position without always adequately reflecting the health needs, wishes and circumstances of each individual child/young person. Whilst some IHA assessments were well completed in very uncertain and challenging circumstances, others were incomplete and lacked robust arrangements for the inclusion of a physical assessment of the child. For this reason, telephone or video-consultations should not replace face-to-face consultations. Proper planning of IHA services and resourcing for recovery is of paramount importance for every professional involved. The child's individual welfare and best interests much be central to decision making.

\section{15Q11.2 MICRO-DELETION ASSOCIATED WITH ADHD: TWO CASE REPORTS AND LITERATURE REVIEW}

${ }^{1}$ Hani Ayyash, ${ }^{2}$ Joseph A Abulail, ${ }^{3}$ Hrizantema Dobreva-Chaban, ${ }^{4}$ Michael 0 Ogundele, ${ }^{5}$ Sophie Hurrell, ${ }^{5}$ Sophie Hurrell. ${ }^{1}$ Southend University Hospital NHS Foundation Trust; ${ }^{2}$ Faculty of Medicine, The Hashemite University, Zarqa, Jordan; ${ }^{3} 1$ )Integrated Department of Paediatrics, Mid and South Essex University Hospitals Group, University Hospital NHS Foundation Trust, Southend-on-Sea, UK; ${ }^{4}$ Department of Community Paediatrics, Bridgewater Community Healthcare NHS Foundation Trust, Halton District, UK; ${ }^{5}$ Integrated Department of Paediatrics, Mid and South Essex University Hospitals Group, University Hospital NHS Foundation Trust, Southend-on-Sea, UK

10.1136/archdischild-2021-rcpch.862 
Background Due to an increased awareness of the significance of genetics in neurodevelopmental disorders (NDD) such as Attention Deficit/Hyperactivity Disorder (ADHD) and autism Spectrum Disorder (ASD), there is an ever-increasing discovery of rare genetic mutations in these patients that are associated risk factors for these NDDs.

$15 \mathrm{q} 11.2$ micro-deletion is a very rare genetic variant where affected patients are likely to require support with learning, have an element of speech delay and/or neuropsychiatric disorders such as ASD and ADHD. It is often due to a de novo mutation but it can also be inherited unknowingly due to variable expressivity.

Genetic chromosomal microarray testing has become increasingly requested for patients with 'unexplained developmental delay/intellectual disability, autism spectrum disorders or multiple congenital anomalies.

Objectives We aimed to describe the clinical, behavioural and learning profile of two patients with $15 \mathrm{q} 11.2$ micro-deletion among the cohort of children and young people (CYP) diagnosed with ADHD in a large Teaching Hospital in the South East of England.

Methods We conducted a search in our hospital electronic database for ADHD patients to identify the occurrence of any associated genetic Copy Number Variants (CNV), specifically looking at the presence of the $15 \mathrm{q} 11.2$ micro-deletion. We also carried out a literature review of the latest research on the association between ADHD and $15 \mathrm{q} 11.2$ micro-deletion using several databases including the OVID, EMBASE, CINHAL and Cochrane's Databases.

Results We identified two ADHD patients with 15q11.2 BP1BP2 micro-deletion, also known as Burnside-Bulter Syndrome. Both of our patients also had learning difficulties with unremarkable neonatal backgrounds. Patient A has additional neuro-developmental features and is currently under assessment for possible ASD. He also presents with mild facial dysmorphic features, which has been reported in 92 out of 200 individuals.

Patient B was a looked-after child due to neglect in infancy.

The published literature regarding $15 \mathrm{q} 11.2$ micro-deletion showed wide variation in the reported signs and symptoms among the affected individuals. Most of the papers reported small case series and the article with the highest number of reported cases included 200 individuals. There are a number of individuals that do not have any apparent signs or symptoms. The most commonly reported features include neurological dysfunction, developmental delay (especially with speech and language and motor delay) and neuropsychiatric disorders such as Autism spectrum disorder and Attention deficit hyperactivity disorder. De novo mutations are reported to account for approximately $5 \%$ to $22 \%$ of all $15 \mathrm{q} 11.2$ micro-deletions. There is also a phenotypic overlap with both Prader-Willi and Angelman syndromes.

Conclusions $15 \mathrm{q} 11.2$ micro-deletion is proving to be one of the 'most common cytogenetic abnormalities' in patients with neurodevelopmental disorders and intellectual impairment. More data would be required to investigate its true significance and value in microarray analysis for all patients referred for ADHD and/or ASD assessment.

Clinicians should consider routine assessment for CNVs in CYP with ADHD, especially if they have other multiple comorbid neurodevelopmental disorders and or dysmorphism.

\section{AN AUDIT OF OUR ADHD SERVICE TO REVIEW ASSESSMENTS BEFORE AND AFTER STARTING ADHD MEDICATION AND TO CHECK COMPLIANCE WITH NICE GUIDELINES}

Chinnaiah Yemula, Kathryn Cope. Cambridgeshire Community Services NHS Trust

\subsection{6/archdischild-2021-rcpch.863}

Background Attention Deficit and Hyperactivity Disorder (ADHD) is a common neurodevelopmental condition seen in $5 \%$ of school age children. Children who are commenced on ADHD medication require regular assessments to ensure safety and continuation of medication and to check progress of their condition.

Objectives We would like to ascertain whether:

- baseline cardiovascular assessment and measurements (height, weight, blood pressure and heart rate) have been carried out on children and young people prior to starting ADHD medication

- the initial assessment and subsequent follow ups met the criteria outlined within NICE guidance (2019)

- there are any safeguarding concerns and if present, escalated appropriately

Methods The sample of children and young people was obtained randomly via SystmOne (electronic record) using the read code 'Attention deficit hyperactivity disorder' to identify them between January to December 2019. They were all started on one of the following medications: Medikinet, Medikinet XL, Matoride XL, Concerta XL, Equasym XL and Elvanse. They were assessed and reviewed by an array of different clinicians.

The audit was reviewed against NICE guidance: Attention Deficit Hyperactivity Disorder: Diagnosis and Management (2019).

Results 20 children and young people were included in the audit. All (100\%) had a baseline height, weight, blood pressure and heart rate $(\mathrm{H}, \mathrm{W}, \mathrm{BP}$ and $\mathrm{HR})$ taken and recorded at their initial assessment. 95\% of the children had the baseline readings of their $\mathrm{H}, \mathrm{W}, \mathrm{BP}$ and $\mathrm{HR}$ documented on their clinic letter, and 75\% had their $\mathrm{BP}$ and $\mathrm{HR}$ percentiles recorded.

As per NICE guidance, children on ADHD medication require a repeat blood $\mathrm{BP}$ and $\mathrm{HR}$ after each dose change and every six months. $25 \%$ of the children in this audit had a repeat $\mathrm{BP}$ and $\mathrm{HR}$ within six months, associated with a medication change or dose adjustment. Similarly, NICE advises that children should have a follow up height every 6 months and a follow up weight every 3 months for children under the age of years and every 6 months for children over the age of 10 years. $20 \%$ of children in this audit had a repeat height and weight within these specified timeframes, and this was also associated with a medication change or dose adjustment.

NICE guidance states that all children should have a cardiovascular assessment prior to starting ADHD medication; this audit found that $85 \%$ of children had documentation pertaining to 'normal heart sounds/heart murmur', and the ADHD pre-drug checklist was completed in $80 \%$ of cases.

Safeguarding was also included in this audit, however only $15 \%$ had current or recent safeguarding needs and none of these required any further escalation. 